Disclosure of Interests: Ambre HITTINGER: None declared, Jean-Hugues Salmon Speakers bureau: Janssen Novartis, jean paul eschard: None declared, Isabelle CHARLOT-LAMBRECHT: None declared DOI: 10.1136/annrheumdis-2019-eular.3033

\section{FRI0492 CALCULATING FRAX SCORE IN CLINICAL PRACTICE: PITFALLS AND PROBLEMS}

Navneet Kaur ${ }^{1}$, Barbara Mendez ${ }^{1}$, Avneet Vig ${ }^{2}$, Beverly Johnson ${ }^{1}$, Tony Francis ${ }^{3}$. ${ }^{1}$ Albert Einstein College of Medicine/Jacobi Medical Center, Rheumatology, Bronx, United States of America; ${ }^{2}$ Cleveland Clinic Martin Health, Rheumatology, Port St. Lucie, United States of America; ${ }^{3}$ Christiana Hospital, Nuclear Medicine, Newark, United States of America

Background: Osteoporosis related fractures cause significant morbidity and mortality. FRAX score uses clinical risk factors and country-specific data in addition to Bone Mineral Density (BMD) to assess patients with high 10-year risk of hip $(>3 \%)$ or major osteoporotic $(>20 \%)$ fracture. We noticed discrepancies between radiologist reported and physician calculated FRAX scores at our hospital. We hypothesized that providers are calculating FRAX score differently as BMD in the FRAX calculator is an optional input variable.

Objectives: This study was initiated to see the differences in results when FRAX score is calculated using T-score, BMD and no BMD and how this difference can influence treatment.

Methods: Retrospective chart review of 1200 DEXA reports from 2013 to 2015 was done. Patients between ages of 40-90 years with T-score ranging from $\leq-1$ to $>-2.5$ at femoral neck were included in the study. Patients already on osteoporosis therapy and/or with T-score $\leq-2.5$ or $>-$ 1 were excluded.

Pertinent risk factors were obtained from chart review. 237 patients met inclusion criteria and 3 separate FRAX scores were calculated using a) Femoral neck BMD b) T-score c) no BMD value. FRAX score reported by radiologist in the chart was also reviewed. Subsequently, FRAX scores obtained using T-score, no BMD value and radiology reported scores were compared against FRAX score calculated using femoral neck BMD (gold standard)

Results:

Table 1. shows demographic information for 237 patients who met inclusion criteria.

\begin{tabular}{lc}
\hline Demographics & \\
\hline Race & \\
Hispanic & $131(54.8 \%)$ \\
Black & $71(29.9 \%)$ \\
Caucasian & $15(6.3 \%)$ \\
Asian & $20(8.5 \%)$ \\
Average Age & $67 \pm 10.5$ years \\
Sex & \\
Male & $11(4.7 \%)$ \\
Female & $226(95.3 \%)$ \\
Average Height & $159.3 \pm 7.6 \mathrm{~cm}$ \\
Average Weight & $71 \pm 13 \mathrm{~kg}$ \\
BMl & $27.9 \pm 4.9$ \\
Previous fracture & $13(5.4 \%)$ \\
Current smoking & $14(5.9 \%)$ \\
Steroid use & $48(20.3 \%)$ \\
RA & $26(11 \%)$ \\
Secondary osteoporosis & $8(3.4 \%)$ \\
Alcohol use & $3(1.3 \%)$ \\
\hline
\end{tabular}

When FRAX score calculated using BMD was compared with FRAX score calculated without BMD, number of patients with high 10-year fracture probability decreased from 49 to 11 patients, which was a statistically significant decrease of $77.6 \%(p<0.001)$.

When data was stratified according to age, there was significant overestimation of risk in patients $>65$ years $(p<0.0001)$ when FRAX was calculated without BMD.

Conclusion: FRAX score calculation without BMD leads to both statistically and clinically significant overdiagnosis especially in elderly.

Interchanging $\mathrm{T}$ score and BMD to calculate FRAX score leads to same treatment decision.

Further education of providers regarding FRAX score is needed.

Many providers are not aware that if BMD column is left blank, it defaults the calculation to no BMD. A pop up alerting the user "no machine was selected so the calculation will default to no BMD which can lead to overestimation of risk" in FRAX tool might be helpful to avoid miscalculation.
REFERENCES:

[1] https://www.sheffield.ac.uk/FRAX/tool.jsp

[2] Prawiradilaga, RS, et al., "FRAX Calculated without BMD Resulting in a Higher Fracture Risk Than That Calculated with BMD in Women with Early Breast Cancer," Journal of Osteoporosis, vol. 2018, Article ID 4636028.

Table 2. shows difference in risk estimation when different FRAX score assessments were compared against gold standard femoral neck BMD.

\begin{tabular}{|c|c|c|c|c|}
\hline $\begin{array}{l}\text { FRAX Scores } \\
\text { Compared }\end{array}$ & $\begin{array}{c}\text { Discrepancy } \\
\text { in } \\
\text { Risk } \\
\text { estimation }\end{array}$ & Overestimation & Underestimation & $\begin{array}{c}\text { McNemar's } \\
\text { test }\end{array}$ \\
\hline $\begin{array}{l}\text { No BMD vs. BMD } \\
\text { FRAX }\end{array}$ & $52 / 237(16 \%)$ & 52 & 0 & $p<0.0001$ \\
\hline $\begin{array}{l}\text { Reported FRAX vs. } \\
\text { BMD } \\
\text { FRAX }\end{array}$ & $6 / 237(2.4 \%)$ & 2 & 4 & $\mathrm{p}=0.414$ \\
\hline $\begin{array}{l}\text { T-score vs. BMD } \\
\text { FRAX }\end{array}$ & $1 / 237(0.4 \%)$ & 1 & 0 & $\mathrm{p}=0.317$ \\
\hline
\end{tabular}

Disclosure of Interests: Navneet Kaur: None declared, Barbara Mendez: None declared, Avneet Vig: None declared, Beverly Johnson Shareholder of: ownership of johnson and johnson stock over 10,000 USD, Consultant for: I am a consultant for the rheumatology education group, Employee of: I have been paid indirectly by pharma as a consultant for the rheumatology education group, Tony Francis: None declared DOI: 10.1136/annrheumdis-2019-eular.1462

\section{FRI0493 CLINICLAL EFFICACY OF DENOSUMAB IN PATIENTS WITH OSTEOPOROSIS BETWEEN RHEUMATOID ARTHRITIS AND PRIMARY OSTEOPOROSIS; 24 MONTHS OF FOLLOW-UP}

Yasuhide Kanayama ${ }^{1}$, Taichi Tsuji ${ }^{2}$, Naohisa Futamura ${ }^{2}$, Naoaki Osada ${ }^{2}$, Hiroto Tachi ${ }^{2}$, Kazunori Todoroki ${ }^{2}$, Ryosuke Sugimoto2. ${ }^{1}$ Toyota Kosei Hospital, Orthopedic Surgery and Rheumatology, Toyota, Japan; ${ }^{2}$ Toyota Kosei Hospital, Orthopedic Surgery, Toyota, Japan

Background: Denosumab (DMB) is a fully human monoclonal antibody to the receptor activator of nuclear factor-kappaB ligand (RANKL) that blocks its binding RANK, inhibiting the development and activity of osteoclasts, decreasing bone resorption, increasing bone density and reducing fracture risk. Also $\mathrm{DMB}$ is a useful therapeutic agent for both rheumatoid arthritis (RA-OP) and primary osteoporosis (P-OP). However there is still few comparative study of clinical efficacy of DMB between RA-OP and P-OP. Objectives: To compare the clinical efficacy of $\mathrm{DMB}$ in patients with osteoporosis between rheumatoid arthritis and primary osteoporosis for 24 months.

Methods: RA patients diagnosed according to the 2010 ACR/EULAR crite ria. RA-OP and P-OP patients met at least one of the following criteria were eligible; a bone mineral density $\mathrm{T}$ score of $-2,5$ or less at the lumber spine or total hip and either one or more moderate or severe vertebral fractures or two or more mild vertebral fractures. All patients were initiated DMB from between October, 2013 and August, 2016. The final study cohort of 61 RA- OP and 50 P-OP patients received continuous DMB therapy more than 24 months. The DMB dose was $60 \mathrm{mg}$ at once every 6 months. In all cases native or activated vitamin $D$ has been used. We reviewed the results for 24 months about the increase and decrease of bone mineral density(BMD) of lumbar spine(LS) and total hip (TH) by DEXA and bone turnover markers, intact n-terminal propeptide type I procollagen(PINP) and tartrate-resistant acid phopshatate form $5 \mathrm{~b}$ (TRACP-5b).

Results: In the patients of RA-OP $(n=61)$ and P-OP $(n=50)$, the number of female was each $56(92 \%)$ and $45(90 \%)$ cases $(p=0.741)$. The mean age was $72.7 \pm 7.5$ and $76.8 \pm 7.8$ years old $(p=0.006)$; disease duration of RA-OP patients was $12.6 \pm 12.9$ years; the body mass index was $20.4 \pm 2.7$ and $20.3 \pm 3.0(p=0.687)$ and the FRAX was $27.2 \pm$ 15.7 and $24.6 \pm 11.1(\mathrm{p}=0.745)$. Clinical findings related to RA-OP at baseline were as follows; CRP $0.8 \pm 1.1$; DAS-CRP $3.28 \pm 1.36$; HAQ $1.16 \pm 1.04$ in RA-OP patients and in the patients of RA-OP and P-OP bone turnover markers and bone mineral density at baseline were as follows; P1NP $57.7 \pm 32.2$ and $64.0 \pm 32.7 \mu \mathrm{g} / \mathrm{l}(\mathrm{p}=0.151) ;$ TRACP-5b 563 